\title{
Direct observation of temperature dependent vortex dynamics in a $\mathrm{La}_{0.7} \mathrm{Sr}_{0.3} \mathrm{MnO}_{3}$ micromagnet
}

\author{
Einar Digernes $\odot,{ }^{1}$ Jonathan Leliaert $\odot,{ }^{2}$ Markus Weigand $\odot,{ }^{3}$ Erik Folven, ${ }^{1}$ and Bartel Van Waeyenberge $\odot^{2, *}$ \\ ${ }^{1}$ Department of Electronic Systems, Norwegian University of Science and Technology, 7491 Trondheim, Norway \\ ${ }^{2}$ Dynamat, Department of Solid State Sciences, Ghent University, 9000 Ghent, Belgium \\ ${ }^{3}$ Helmholtz-Zentrum Berlin für Materialien und Energie GmbH, 14109 Berlin, Germany
}

(Received 8 July 2020; revised 29 October 2020; accepted 7 December 2020; published 28 December 2020)

\begin{abstract}
Although it is well documented that the vortex core in a micromagnet can switch polarization in response to magnetic field pulses, the temperature dependence of this process has not yet been addressed by experiments. Using scanning transmission $\mathrm{x}$-ray microscopy, we investigate the magnetic vortex dynamics in $\mathrm{La}_{0.7} \mathrm{Sr}_{0.3} \mathrm{MnO}_{3}$ microplatelet at temperatures ranging from $150 \mathrm{~K}$ up to $T_{\mathrm{C}}$ at $350 \mathrm{~K}$. The time-resolved images reveal qualitatively different dynamic regimes as a function of temperature and applied field, as the relative strengths of the micromagnetic energy terms strongly vary over this temperature range. By explicitly accounting for the temperature dependence of the magnetic parameters in our micromagnetic simulations, we found excellent agreement with the experiments over the full measurement range. In line with previous models, the simulations reveal that the vortex core switches polarization when it reaches a critical velocity that mainly depends on the strength of the exchange interaction. It is therefore strongly temperature dependent, thus explaining our observation of the different dynamical regimes.
\end{abstract}

DOI: 10.1103/PhysRevResearch.2.043429

\section{INTRODUCTION}

A detailed understanding of the static and dynamic properties of magnetic thin-film structures is crucial for their successful implementation in technological devices such as logic and memory applications [1-3]. One of the most prominent nontrivial configurations is the vortex state. Microstructures defined in soft ferromagnetic thin films can minimize their stray field by adopting a magnetic vortex configuration in which the magnetization is contained in the sample plane and curls around the center of the structure. In square-shaped structures, this state consists of four triangular closure domains and is commonly called the Landau pattern. To avoid the formation of a singularity the magnetization will turn out of the sample plane at the very center, forming the so-called vortex core. In recent years, several schemes have been proposed to exploit the specific dynamics displayed by the vortex and its core in magnetic sensors or memories [4-6]. In addition to potential device applications, the vortex structure is a good test bench for fundamental studies of magnetic interactions.

According to the equation of motion for magnetic structures, introduced by Thiele [7] and applied to vortex structures by Huber [8], a moving vortex core experiences an in-plane gyro force perpendicular to its velocity and with a sign given by the magnetization direction of the core, called the core polarization $p$. This gives rise to a counterclockwise or clock-

\footnotetext{
*bartel.vanwaeyenberge@ugent.be

Published by the American Physical Society under the terms of the Creative Commons Attribution 4.0 International license. Further distribution of this work must maintain attribution to the author(s) and the published article's title, journal citation, and DOI.
}

wise gyration for $p=1$ or $p=-1$, respectively. When a vortex is displaced from its equilibrium position, the restoring force due to the demagnetizing fields will, together with the gyro force, result in gyration around the equilibrium position. This corresponds to the fundamental excitation mode of the vortex structure. With a resonance frequency typically in the subgigahertz range, such a vortex gyration can be directly excited by an alternating magnetic field. This mode was first observed more than 30 years ago [9], but recent advances in magnetic microscopy have revealed unexpected details.

Static vortex core switching in soft magnetic materials requires relatively high magnetic fields on the order of $0.2 \mathrm{~T}$ [10]. However, a dynamic switching process which requires much lower fields also exists [11]. A moving vortex core deforms and develops an area with opposite polarization in its wake (a dip) [12]. At some critical velocity, this dip ultimately evolves into a fully out-of-plane magnetized area, and a vortex-antivortex pair is created. The antivortex then annihilates with the original vortex [11] and leaves behind the new vortex with opposite polarization. Since the discovery of the basic dynamic properties of vortices, i.e., the gyrotropic resonance $[7,13]$ and the vortex core switching dynamics $[11,14]$, the vortex state has been extensively studied in soft magnetic platelets (e.g., NiFe permalloy).

Few data exist on the vortex dynamics in epitaxial materials. Thin films of the ferromagnetic complex oxide $\mathrm{La}_{0.7} \mathrm{Sr}_{0.3} \mathrm{MnO}_{3}$ (LSMO) have a Curie temperature close to room temperature $\left(T_{\mathrm{C}}=350 \mathrm{~K}\right)$ and a pronounced magnetocrystalline anisotropy. The relative strengths of the magnetostatic, exchange, and anisotropy energies are strongly temperature dependent close to room temperature, making LSMO an excellent model system for investigating temperature-dependent magnetization dynamics. However, as the substrates used (e.g., $\mathrm{SrTiO}_{3}$ ) are not transparent to soft $\mathrm{x}$ 
(a)

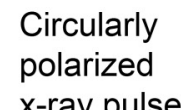

x-ray pulses

aperture

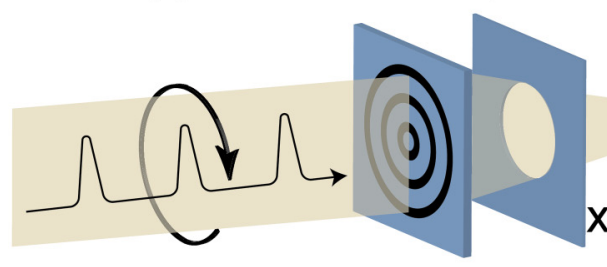

(c)

(d)
Zone Order Sample Photodetector

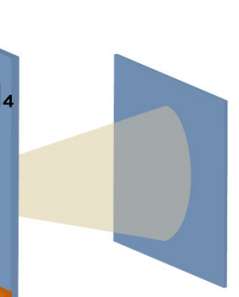

(b)

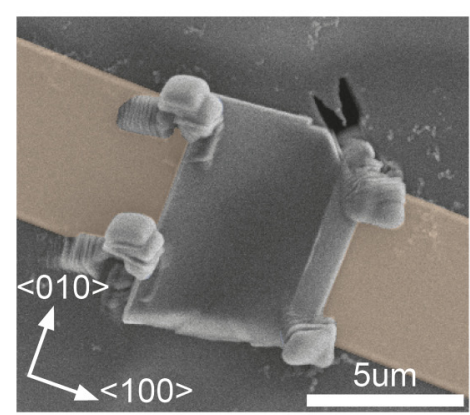

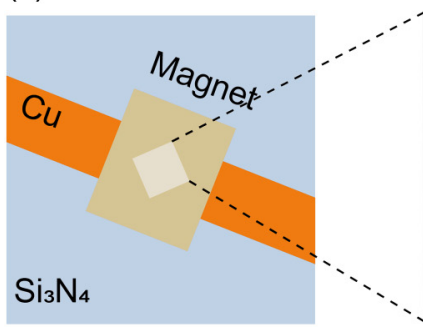

$T=261 \mathrm{~K}$
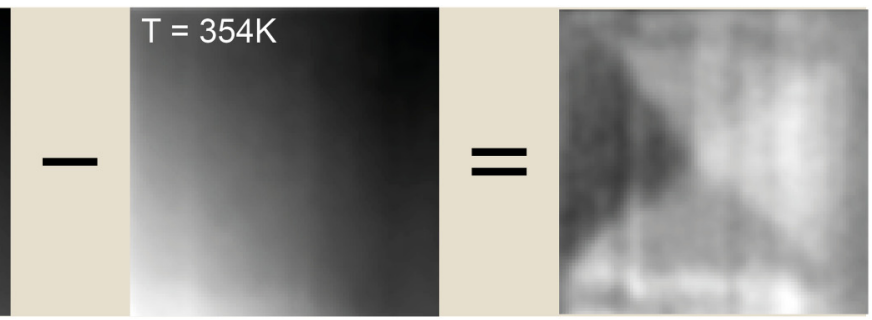

FIG. 1. (a) Schematic of the scanning electron microscope setup. (b) and (c) Scanning electron micrograph and schematic of the lamella (beige) on top of the stripline. (d) Images illustrating subtracting the nonmagnetic background captured above $T_{\mathrm{C}}=350 \mathrm{~K}$ and the resulting magnetic contrast image.

rays, they must be thinned to only a few hundred nanometers thick to be compatible with scanning transmission $\mathrm{x}$-ray microscopy (STXM) investigations [15].

Here, we use STXM to investigate an epitaxial LSMO micromagnet in a lamella extracted by a focused ion beam (FIB). This enables us to study the vortex dynamics in a temperature range from $150 \mathrm{~K}$ up to the Curie temperature. The micromagnet is excited by magnetic field pulses from a stripline. We report the direct observation of qualitatively different dynamical regimes as a function of temperature.

\section{METHODS}

An epitaxial thin film of LSMO was grown on a (001)oriented 0.05 wt \% Nb-doped $\mathrm{SrTiO}_{3}$ as described in [16]. In situ reflection high-energy electron diffraction and subsequent x-ray diffraction measurements revealed a 43-nm (112 unit cells) thick film of high crystalline quality that was fully strained to the $\mathrm{SrTiO}_{3}$ (STO) substrate. An embedded $2 \times 2 \mu \mathrm{m}^{2}$ square micromagnet was defined using electron beam lithography and $\mathrm{Ar}^{+}$ion implantation to locally disrupt the magnetic order [17,18], leaving ferromagnetic squares in a paramagnetic matrix. The sides of the squares were along $\langle 110\rangle$ directions, corresponding to the magnetocrystalline easy axes of the LSMO thin film under tensile strain from the (001)-oriented $\mathrm{SrTiO}_{3}$ substrate [19]. To allow for imaging using STXM, a lamella containing one square micromagnet was cut out using a DualBeam FIB scanning electron microscope. Prior to the thinning of the sample with FIB, a protective carbon layer was deposited in situ to shield the film from ion-beam-induced damage. Similar approaches are routinely used for preparation of transmission electron microscopy samples and have been shown to induce minimal damage in LSMO thin films thinned down to $120 \mathrm{~nm}$ [20].
Subsequently, it was transferred to a 150-nm-thick and 5 - $\mu$ m-wide $\mathrm{Cu}$ stripline patterned on a soft $\mathrm{x}$-ray transparent 150-nm-thick $\mathrm{Si}_{3} \mathrm{~N}_{4}$ membrane (see Fig 1). The lamella was oriented such that in-plane magnetic field pulses could be applied along the $\langle 010\rangle$ diagonal of the square magnet by passing a current through the stripline. For further details see the Supplemental Material [21]. The sample is mounted on a cryostat enabling cooling down to $150 \mathrm{~K}$.

The magnetization dynamics of the micromagnet were captured by measuring the transmittance of circularly polarized $\mathrm{x}$ rays tuned to the $\mathrm{Mn} L_{3}$ absorption edge $\left(E_{L_{3}}=642.8 \mathrm{eV}\right)$. $\mathrm{X}$-ray magnetic circular dichroism causes the absorption to depend on the orientation of the $\mathrm{x}$-ray polarization vector relative to the magnetization vector in the sample [22]. This mechanism provides magnetic contrast in the obtained images. The $\mathrm{x}$ rays were focused to a spot size below $20 \mathrm{~nm}$ using a zone plate and were incident at a $30^{\circ}$ angle to the surface normal in order to be sensitive to in-plane magnetization. The STXM microscope at the MAgnetic X-raY Microscope with UHV Spectroscopy (MAXYMUS) beamline at Bessy II was used in this work [23].

Time resolution is obtained by acquiring images in a stroboscopic manner. The sample is excited with magnetic field pulses with a controlled delay with respect to the x-ray flashes. This allows capturing the magnetization dynamics one pixel at a time with a time resolution equal to the 35-ps length of the $\mathrm{x}$-ray flashes. As the recorded images are accumulated over several minutes, they show the dynamics averaged over a large number of excitations.

When applying field pulses, Joule heating in the stripline gave rise to a temperature increase. This was observed by an increase in stripline resistance and reduction of magnetic contrast. The sample temperature was estimated based on the temperature measured on the sample holder before application 
(a)

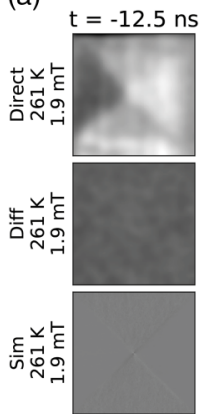

(b)

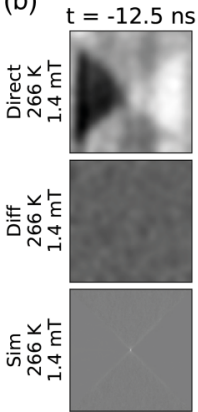

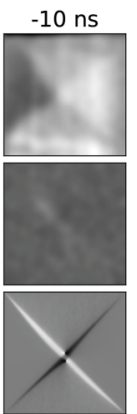

$-10 \mathrm{~ns}$

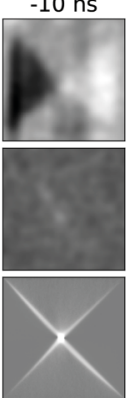

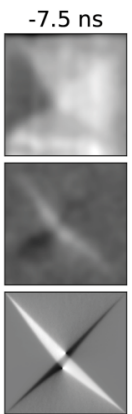

$-7.5 \mathrm{~ns}$

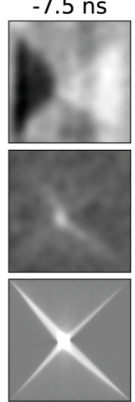

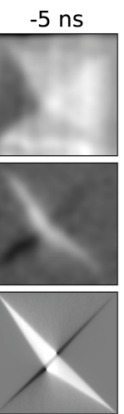

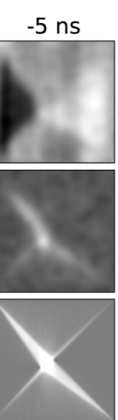

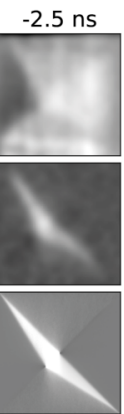

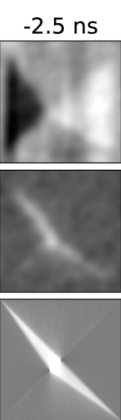

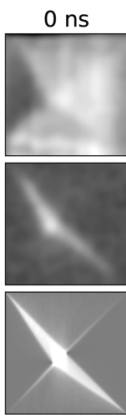

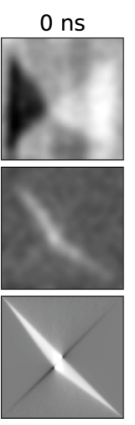

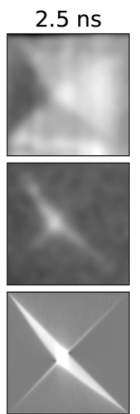

$2.5 \mathrm{~ns}$

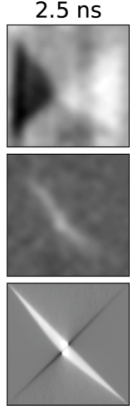

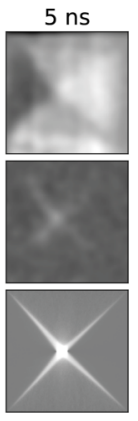

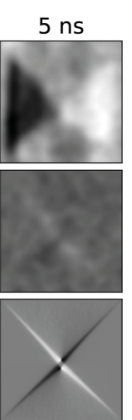

(c)
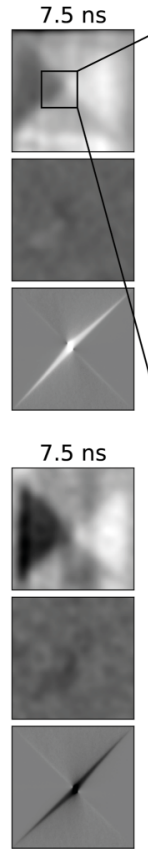

(d)
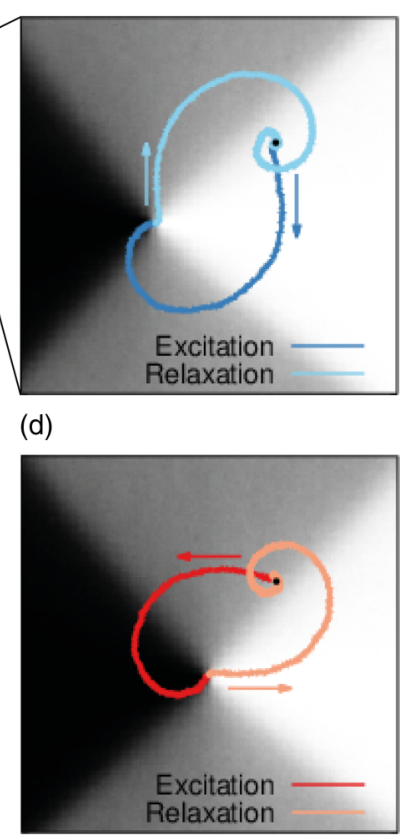

FIG. 2. Time-resolved STXM data and simulation of magnetization dynamics for (a) 1.9-mT field pulses at $261 \mathrm{~K}$ and (b) $1.4-\mathrm{mT}$ field pulses at $266 \mathrm{~K}$. In (a) and (b) the top row displays direct images after subtracting the background, the middle row is the difference from the nonexcited ground state, and bottom row is the corresponding simulated difference series. The images shown in (a) and (b) are $2 \times 2 \mu \mathrm{m}^{2}$. Vortex core trajectory from simulations displays (c) clockwise and (d) counterclockwise gyration, corresponding to negative and positive core polarization, respectively. The background shows the average magnetization state at the end of the excitation, while the black dot shows the equilibrium vortex core position in the absence of external fields. (c) and (d) depict a $0.6 \times 0.6 \mu \mathrm{m}^{2}$ region near the center of the micromagnet.

of the field pulses and the resistance change of the stripline, as detailed in the Supplemental Material [21].

Simulations are performed using the GPU-accelerated [24] micromagnetic software package MUMAX3 [25], which solves the Landau-Lifshitz-Gilbert equation. The simulations take into account the temperature dependence of the saturation magnetization $M_{\mathrm{s}}$, the magnetocrystalline anisotropy $K$ [26], and the exchange constant $A_{\text {ex }}[27,28]$. To allow a visual comparison to the recorded STXM images (Figs. 2 and 3), their numerical counterparts are simulated for (and averaged over) 20 consecutive field pulse cycles. Here, thermal fluctuations are added and time integrated using an adaptive time stepping algorithm [29]. The simulations results presented in Fig. 4, are integrated over five consecutive field pulse cycles. For further details on the micromagnetic simulations, see the Supplemental Material [21].

\section{RESULTS}

The magnetic vortex core gyration in the Landau pattern is excited by application of 12-ns magnetic field pulses along (a)

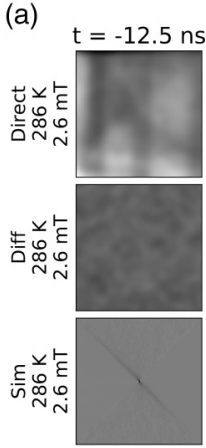

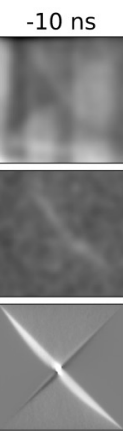
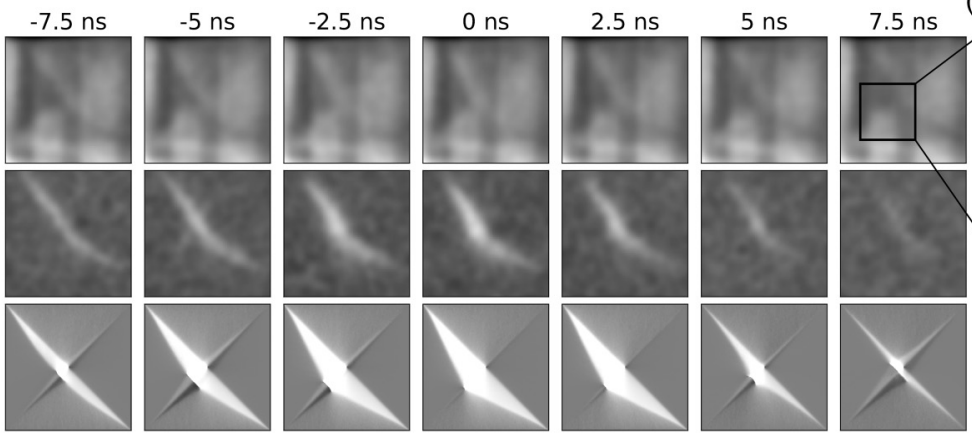

(b)

FIG. 3. Time-resolved STXM data and simulations of magnetization dynamics for 2.6-mT field pulses at $286 \mathrm{~K}$. (a) The top row displays direct images after subtracting the background, the middle row is the difference from the nonexcited ground state, and the bottom row is the simulated difference series. The images shown are $2 \times 2 \mu \mathrm{m}^{2}$. (b) Characteristic vortex core trajectories from the same simulation shown for a $0.9 \times 0.9 \mu \mathrm{m}^{2}$ region near the center of the micromagnet. The background shows the average magnetization state at the end of the excitation, while the black dot shows the equilibrium vortex core position in the absence of external fields. 


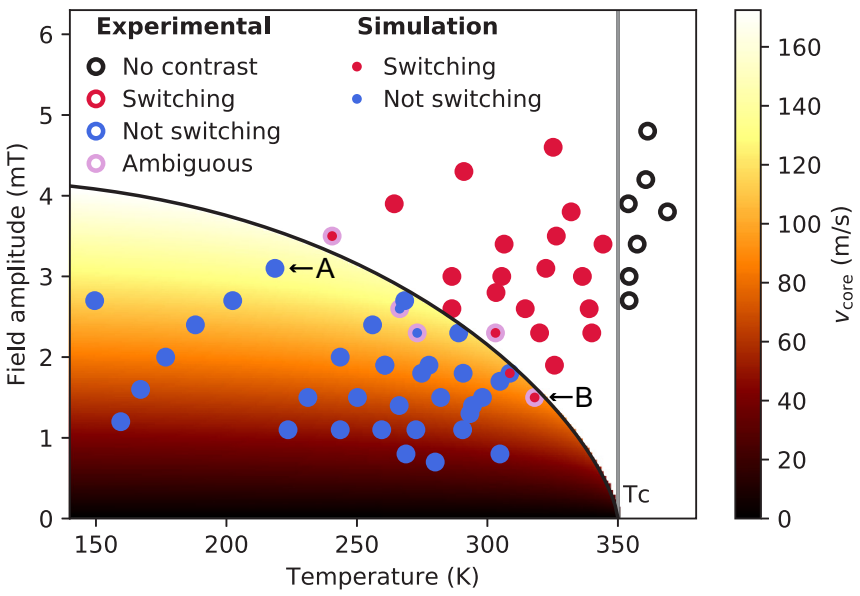

FIG. 4. Classification of dynamics for experimental data points (edge color) and simulations (center color). The data point with the highest field amplitude without switching is labeled A, and the data point with the lowest field amplitude with switching is labeled $\mathrm{B}$. The background is the estimated maximum vortex core velocity $v_{\text {core }}$ for excitations up to the critical field (solid black line) calculated with Eq. (2).

the diagonal of the square every $40 \mathrm{~ns}$. The zero point in time, $t=0 \mathrm{~ns}$, is set to the end of the excitation pulse. The resulting dynamics for 1.9-mT field amplitude recorded at $261 \mathrm{~K}$ are displayed in Fig. 2(a). The top row shows direct images where the nonmagnetic background, mainly from the nonuniform substrate, has been subtracted, as exemplified in Fig. 1(d). In the second row a differential time series is calculated as the difference from the nonexcited ground state. Contrast in the differential series thus corresponds to movement of domain walls and the vortex core. When the field is applied, the vortex core gyrates towards the new equilibrium position. The diagonal "cross" that appears in the differences series at $t=-7.5 \mathrm{~ns}$ is the signature of a clockwise gyration during the excitation. The black line disappears at $t=-2.5 \mathrm{~ns}$ and reappears as a white line at $t=2.5 \mathrm{~ns}$, corresponding to clockwise gyration during relaxation. Thus, the distinct black and white lines and the switching between them is the signature of gyration without switching of the polarization. The results are complemented by micromagnetic simulations, shown in the bottom row, where the vortex core is initialized with $p=-1$.

The observed dynamics closely resemble the experimental data. The vortex core trajectory is plotted from the simulated data in Fig. 2(c). The data show clockwise gyration on both excitation and relaxation and no switching of the vortex core polarization. See Supplemental Movie 1 [21].

Figure 2(b) displays dynamics resulting from a field excitation amplitude of $1.4 \mathrm{mT}$ recorded at $266 \mathrm{~K}$. The difference series show a white diagonal cross on the excitation which corresponds to the opposite gyration handedness from Fig. 2(a), resulting from a core polarization reversal in between these measurements. Simulations with $p=+1$ agree with the experiment and show counterclockwise gyration without vortex core switching. See Supplemental Movie 2 [21].

Figure 3(a) displays the dynamics for a field amplitude of $2.6 \mathrm{mT}$. In contrast to Fig. 2, it is not possible to determine a specific handedness of the gyration. This suggests that the vortex core polarization is switching. In this case, the recorded data are a superposition of the different vortex core trajectories. Micromagnetic simulations mirroring the experiment show switching of the polarization and that the number of switching events varies from one pulse to the next. Four observed vortex core trajectories are plotted in Fig. 3(b). See Supplemental Movie 3 [21].

The magnetization dynamics have been recorded experimentally at temperatures ranging from 150 to above $350 \mathrm{~K}$ and with field pulses of 12-ns duration and with an excitation amplitude up to $5 \mathrm{mT}$. Because the upper boundary of this temperature range coincides with the Curie temperature, the materials parameters, and the relevant strength of the resulting micromagnetic energy terms, rapidly change in this interval. As the Curie temperature is approached, we observe a gradual decrease in magnetic contrast due to the reduced saturation magnetization.

To gain further insight into the vortex dynamics, the experimental conditions have been used as inputs to micromagnetic simulations. Each simulation is run for five consecutive magnetic field pulses, and the vortex core polarization, its trajectory, and its velocity are tracked.

In Fig. 4, the experimental and simulation results are classified based on the observed dynamics: the data points are given different colors at the edge (experiment) and center (simulations). A data point where the center and the edge are the same color indicates agreement between simulation and experiment. Measurements where a single handedness of the gyration is observed, as depicted in Fig. 2, are labeled as not switching (blue). Image series with the signature of vortex core switching, similar to the one displayed in Fig. 3, are classified as switching (red). In addition to these two main classifications, we have experimental data points that are too ambiguous to classify in one of the former categories (purple) or that show no magnetic contrast at all (black).

The classification results in three distinct regions in the diagram shown in Fig. 4. To the lower left, in the region of low temperature and field, the vortex core polarization is not switching. When temperature is increased or the field is increased, the vortex core polarization is switching. Thus, there is a temperature-dependent critical field for initiating vortex core switching. Finally, the magnetization vanishes above $T_{\mathrm{C}}$, and no magnetic signal is observed above this temperature.

All data points except six are classified the same way in experiment and simulation. Five of them are classified as ambiguous in the experiment and are not necessarily in disagreement with the simulations. One data point, close to the phase boundary, is switching in the simulation and not in the experiment. Generally, however, we note that there is excellent agreement between experiment and simulation over the wide range of experimental conditions. Here, we emphasize that for the simulations, all temperature dependent material parameters are taken from the literature or from independent measurements on our sample, while the field excitation mimics the experiment as closely as possible. Therefore, the agreement between simulation and experiment was not aided by the tuning or fitting of these parameters. Consequently, these simulations offer a unique and reliable tool to understand the temperature and field dependence of 


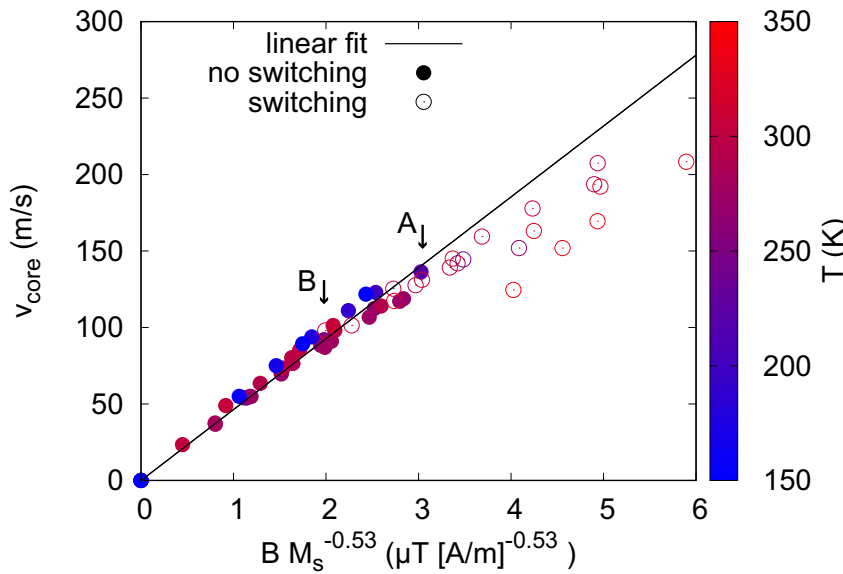

FIG. 5. Maximum velocity attained by the vortex core as extracted from the micromagnetic simulations, mirroring the experiments. The maximum velocity is shown as a function of the applied field amplitude $B$, rescaled with saturation magnetization $M_{s}$ raised to the power -0.53 , which is a phenomenological constant which makes all data fall onto a single line. The slope of this line can be used to estimate the maximum core velocity for arbitrary field and temperature, as used in the background of Fig. 4. The temperatures are given by the colors of the data points. Note that there is no trivial temperature dependence of the maximum core velocity. The linear fit was made to all data points that do not show switching (the blue data points in Fig. 4). The data point with the highest core velocity without switching is labeled $\mathrm{A}$, and the data point with the lowest core velocity with switching is labeled B.

the phase boundary between the switching and nonswitching regions in our data.

\section{DISCUSSION}

Guslienko et al. [30] showed that a moving vortex gives rise to a dynamic out-of-plane effective field resulting in the core deformation and, ultimately, a vortex antivortex pair creation. As this dynamic field is proportional to the vortex velocity, this model allows for the estimation of critical velocity at which this pair is created and the core reversal process is initiated.

The resolution of the experimental measurement data does not allow us to determine the core velocity accurately. We therefore extract it from the simulations mirroring the experimental data. The maximum velocity attained by the vortex core throughout each simulation $v_{\text {core }}$ is presented in Fig. 5. The solid data points display the maximum core velocity in simulations that do not display any core switching (blue points in Fig. 4). As shown in Fig. 5, all data points fall on a straight line given by $v_{\text {core }}=47.3 \times 10^{6} B M_{s}^{-0.53}$.

The open circles, corresponding to the simulations that do display vortex core switching, lie below the line. This is due to the fact that part of the energy dissipates in the switching process, thus resulting in a lower maximum velocity.

The maximum core velocity is visualized as the colored background in Fig. 4 based on the linear fit shown in Fig. 5. The velocity at the boundary between the switching and nonswitching data points is strongly temperature dependent. We do not observe switching at $220 \mathrm{~K}$ for a velocity of $136 \mathrm{~ms}$ (data point $\mathrm{A}$ in Figs. 4 and 5), whereas switching takes place at less than $98 \mathrm{~ms}$ at $320 \mathrm{~K}$ (data point $\mathrm{B}$ ). These values are significantly below the value reported in the literature of $330 \mathrm{~ms}$ for permalloy [31,32] and predictions above $400 \mathrm{~ms}$ for $\mathrm{Fe}$ and $\mathrm{Co}[32]$ at room temperature.

In the phenomenological model presented by Lee et al. [32] the critical velocity value for soft magnetic materials is given by

$$
v_{\text {crit }}=(1.66 \pm 0.18) \gamma \sqrt{\frac{\mu_{0} A_{\mathrm{ex}}}{4 \pi}} .
$$

In this equation, $\mu_{0}$ and $\gamma$ denote the vacuum permeability and gyromagnetic ratio, respectively. This critical velocity depends on only the exchange parameter as the dynamic effective field [30] has its origin in the exchange interaction [32].

To include the effect of the magnetocrystalline anisotropy of the LSMO samples, we follow the approach presented in Ref. [33], where the presence of an external out-of-plane field $H$ was accounted for by correcting Eq. (1) with a factor $\left(1-H / H_{\text {crit }}\right) . H_{\text {crit }}$ is the static out-of-plane field necessary to switch the vortex core polarization [33]. By considering the anisotropy field $H_{k}=2 K / M_{s}$ as an effective out-of-plane field, with $K$ being the magnetocrystalline anisotropy constant, we arrive at the following expression for the critical core velocity:

$$
v_{\text {crit }}=(1.66 \pm 0.18) \gamma \sqrt{\frac{\mu_{0} A_{\mathrm{ex}}}{4 \pi}}\left(1-\frac{H_{k}}{H_{\text {crit }}}\right) .
$$

As shown in the Supplemental Material [21], we verified with an additional set of simulations that this anisotropy term is necessary to correctly describe the critical velocity down to $0 \mathrm{~K}$. Similar to what is reported in [33] for easy-plane anisotropy, also cubic anisotropy reduces the critical velocity.

The critical velocity $v_{\text {crit }}$ can be transformed into the temperature dependent critical field (black line in Fig. 4) by using the linear fit in Fig. 5 and filling in the temperature dependent parameters for LSMO. The calculated critical velocity excellently describes the boundary between the switching and nonswitching data points over a wide field and temperature range.

\section{CONCLUSION}

We report the direct observation of the field and temperature dependence of the vortex core dynamics in a LSMO microplatelet using scanning transmission $\mathrm{x}$-ray microscopy. We identified the transition from gyration to vortex core switching in a temperature range close to $T_{\mathrm{C}}$, where the relative strengths of the magnetic parameters strongly vary. In the experiments reported here, the critical field amplitude for switching ranges from $3.5 \mathrm{mT}$ at $225 \mathrm{~K}$ down to $0 \mathrm{mT}$ at $T_{\mathrm{C}}$.

These experiments were complemented with matching micromagnetic simulations that consider the temperature variations of the micromagnetic parameters based on independent data. Good agreement with the experimentally observed dynamics was achieved. The micromagnetic model also allowed us to investigate the vortex velocity and demonstrate a strong temperature dependence of the critical velocity. It ranges from $160 \mathrm{~ms}$ at $150 \mathrm{~K}$ to almost $0 \mathrm{~ms}$ close to $T_{\mathrm{C}}$. As predicted by the model, this is mainly the result of a vanishing exchange pa- 
rameter near $T_{\mathrm{C}}$. The considered cubic anisotropy also reduced the critical velocity. As a lower critical velocity coincides with a lower critical excitation strength, facilitating core switching, these results opens ways to search for suitable materials with optimal performance for applications at room temperature. These results also demonstrate that micromagnetics can capture and give more insight into the dynamics over a broad temperature range.

\section{ACKNOWLEDGMENTS}

We thank P. E. Vullum for the valuable input on the FIB processing. We thank HZB for the allocation of synchrotron radiation beam time. This work was supported by the Fonds
Wetenschappelijk Onderzoek (FWO-Vlaanderen) with a postdoctoral fellowship (J.L.). We gratefully acknowledge the support of NVIDIA Corporation with the donation of part of the GPUs used for this research. Part of the computational resources (Stevin Supercomputer Infrastructure) and services used in this work was provided by the VSC (Flemish Supercomputer Center), funded by Ghent University, FWO, and the Flemish government, department EWI. Partial funding was obtained from the Norwegian $\mathrm{PhD}$ Network on Nanotechnology for Microsystems, which is sponsored by the Research Council of Norway, Division of Science, under Contract No. 221860/F60. The Research Council of Norway is acknowledged for support to the Norwegian Micro- and Nanofabrication Facility, NorFab, under Contract No. 245963/F50.
[1] D. A. Allwood, G. Xiong, C. C. Faulkner, D. Atkinson, D. Petit, and R. P. Cowburn, Magnetic domain-wall logic, Science 309, 1688 (2005).

[2] S. Parkin, M. Hayashi, and L. Thomas, Magnetic domain-wall racetrack memory, Science 320, 190 (2008).

[3] A. Fert, N. Reyren, and V. Cros, Magnetic skyrmions: Advances in physics and potential applications, Nat. Rev. Mater. 2, 17031 (2017).

[4] D. Suess, A. Bachleitner-Hofmann, A. Satz, H. Weitensfelder, C. Vogler, F. Bruckner, C. Abert, K. Prügl, J. Zimmer, C. Huber, S. Luber, W. Raberg, T. Schrefl, and H. Brückl, Topologically protected vortex structures for low-noise magnetic sensors with high linear range, Nat. Electron. 1, 362 (2018).

[5] H. Jung, Y.-S. Choi, K.-S. Lee, D.-S. Han, Y.-S. Yu, M.-Y. Im, P. Fischer, and S.-K. Kim, Logic operations based on magneticvortex-state networks, ACS Nano 6, 3712 (2012).

[6] R. Hertel, Vortex states à la carte, Nat. Nanotechnol. 8, 318 (2013).

[7] A. A. Thiele, Steady-State Motion of Magnetic Domains, Phys. Rev. Lett. 30, 230 (1973).

[8] D. L. Huber, Dynamics of spin vortices in two-dimensional planar magnets, Phys. Rev. B 26, 3758 (1982).

[9] B. E. Argyle, E. Terrenzio, and J. C. Slonczewski, Magnetic Vortex Dynamics Using the Optical Cotton-Mouton Effect, Phys. Rev. Lett. 53, 190 (1984).

[10] A. Thiaville, J. M. García, R. Dittrich, J. Miltat, and T. Schrefl, Micromagnetic study of Bloch-point-mediated vortex core reversal, Phys. Rev. B 67, 094410 (2003).

[11] B. Van Waeyenberge, A. Puzic, H. Stoll, K. W. Chou, T. Tyliszczak, R. Hertel, M. Fahnle, H. Bruckl, K. Rott, G. Reiss, I. Neudecker, D. Weiss, C. H. Back, and G. Schutz, Magnetic vortex core reversal by excitation with short bursts of an alternating field, Nature (London) 444, 461 (2006).

[12] A. Vansteenkiste, K. Chou, M. Weigand, M. Curcic, V. Sackmann, H. Stoll, T. Tyliszczak, G. Woltersdorf, C. Back, G. Schütz et al., X-ray imaging of the dynamic magnetic vortex core deformation, Nat. Phys. 5, 332 (2009).

[13] S. Choe, Y. Acremann, A. Scholl, A. Bauer, A. Doran, J. Stohr, and H. Padmore, Vortex core-driven magnetization dynamics, Science 304, 420 (2004).
[14] K. Yamada, S. Kasai, Y. Nakatani, K. Kobayashi, H. Kohno, A. Thiaville, and T. Ono, Electrical switching of the vortex core in a magnetic disk, Nat. Mater. 6, 269 (2007).

[15] J. Förster, S. Wintz, J. Bailey, S. Finizio, E. Josten, C. Dubs, D. A. Bozhko, H. Stoll, G. Dieterle, N. Träger, J. Raabe, A. N. Slavin, M. Weigand, J. Gräfe, and G. Schütz, Nanoscale x-ray imaging of spin dynamics in yttrium iron garnet, J. Appl. Phys. 126, 173909 (2019).

[16] E. Folven, A. Scholl, A. Young, S. T. Retterer, J. E. Boschker, T. Tybell, Y. Takamura, and J. K. Grepstad, Effects of nanostructuring and substrate symmetry on antiferromagnetic domain structure in $\mathrm{LaFeO}_{3}$ thin films, Phys. Rev. B 84, 220410(R) (2011).

[17] Y. Takamura, R. V. Chopdekar, A. Scholl, A. Doran, J. A. Liddle, B. Harteneck, and Y. Suzuki, Tuning magnetic domain structure in nanoscale $\mathrm{La}_{0.7} \mathrm{Sr}_{0.3} \mathrm{MnO}_{3}$ islands, Nano Lett. 6, 1287 (2006).

[18] E. Folven, T. Tybell, A. Scholl, A. Young, S. T. Retterer, Y. Takamura, and J. K. Grepstad, Antiferromagnetic domain reconfiguration in embedded $\mathrm{LaFeO}_{3}$ thin film nanostructures, Nano Lett. 10, 4578 (2010).

[19] L. M. Berndt, V. Balbarin, and Y. Suzuki, Magnetic anisotropy and strain states of (001) and (110) colossal magnetoresistance thin films, Appl. Phys. Lett. 77, 2903 (2000).

[20] K. O'Shea, D. McGrouther, C. Ferguson, M. Jungbauer, S Hühn, V. Moshnyaga, and D. MacLaren, Fabrication of high quality plan-view TEM specimens using the focused ion beam, Micron 66, 9 (2014).

[21] See Supplemental Material at http://link.aps.org/supplemental/ 10.1103/PhysRevResearch.2.043429 for supplemental movies and extra details on the samples and data processing.

[22] G. Schütz, W. Wagner, W. Wilhelm, P. Kienle, R. Zeller, R. Frahm, and G. Materlik, Absorption of Circularly Polarized X Rays in Iron, Phys. Rev. Lett. 58, 737 (1987).

[23] R. Follath, J. S. Schmidt, M. Weigand, and K. Fauth, The X-ray microscopy beamline UE46-PGM2 at BESSY, in SRI 2009, 10th International Conference on Radiation Instrumentation, edited by R. Garrett, I. Gentle, K. Nugent, and S. Wilkins, AIP Conf. Proc. No. 1234 (AIP, Melville, NY, 2010), p. 323.

[24] J. Leliaert, M. Dvornik, J. Mulkers, J. De Clercq, M. V. Milošević, and B. Van Waeyenberge, Fast micromagnetic 
simulations on GPU-recent advances made with mumax3, J. Phys. D 51, 123002 (2018).

[25] A. Vansteenkiste, J. Leliaert, M. Dvornik, M. Helsen, F. GarciaSanchez, and B. Van Waeyenberge, The design and verification of MuMax3, AIP Adv. 4, 107133 (2014).

[26] K. Steenbeck and R. Hiergeist, Magnetic anisotropy of ferromagnetic $\mathrm{La}_{0.7}(\mathrm{Sr}, \mathrm{Ca})_{0.3} \mathrm{MnO}_{3}$ epitaxial films, Appl. Phys. Lett. 75, 1778 (1999).

[27] R. Moreno, R. F. L. Evans, S. Khmelevskyi, M. C. Muñoz, R. W. Chantrell, and O. Chubykalo-Fesenko, Temperaturedependent exchange stiffness and domain wall width in Co, Phys. Rev. B 94, 104433 (2016).

[28] M. S. Lee, T. A. Wynn, E. Folven, R. V. Chopdekar, A. Scholl, A. T. Young, S. T. Retterer, J. K. Grepstad, and Y. Takamura, Tailoring spin textures in complex oxide micromagnets, ACS Nano 10, 8545 (2016).

[29] J. Leliaert, J. Mulkers, J. De Clercq, A. Coene, M. Dvornik, and B. Van Waeyenberge, Adaptively time stepping the stochastic
Landau-Lifshitz-Gilbert equation at nonzero temperature: Implementation and validation in MuMax3, AIP Adv. 7, 125010 (2017).

[30] K. Y. Guslienko, K.-S. Lee, and S.-K. Kim, Dynamic Origin of Vortex Core Switching in Soft Magnetic Nanodots, Phys. Rev. Lett. 100, 027203 (2008).

[31] S.-K. Kim, Y.-S. Choi, K.-S. Lee, K. Y. Guslienko, and D.-E. Jeong, Electric-current-driven vortex-core reversal in soft magnetic nanodots, Appl. Phys. Lett. 91, 082506 (2007).

[32] K.-S. Lee, S.-K. Kim, Y.-S. Yu, Y.-S. Choi, K. Y. Guslienko, H. Jung, and P. Fischer, Universal Criterion and Phase Diagram for Switching a Magnetic Vortex Core in Soft Magnetic Nanodots, Phys. Rev. Lett. 101, 267206 (2008).

[33] A. V. Khvalkovskiy, A. N. Slavin, J. Grollier, K. A. Zvezdin, and K. Y. Guslienko, Critical velocity for the vortex core reversal in perpendicular bias magnetic field, Appl. Phys. Lett. 96, 022504 (2010) 